\title{
Complementarity Formulation for Multi-Fingered Hand Manipulation with Rolling and Sliding Contacts
}

\author{
Masahito Yashima Hideya Yamaguchi \\ Dept. of Mechanical Systems Engineering, \\ National Defense Academy \\ Yokosuka 239-8686, Japan
}

\begin{abstract}
We propose a novel complementarity model for a general three-dimensional manipulation system with rolling and sliding contacts to solve the forward dynamics problem. The key idea is to derive complementary constraints for a rolling contact by decomposing the tangential properties. We also discuss the existence and uniqueness of a solution to the model, and algorithms for solving the problem. We show examples of computer simulation for dynamics of multifingered hand manipulation and discuss how the transition of contact modes varies for applied joint driving torques.
\end{abstract}

\section{INTRODUCTION}

When we consider a manipulation system by a multifingered hand, an object has multiple contact points lying within the hand. Therefore, the object is moved by the multi-fingered hand in response to computer activated commands. Forces between the object and the multifingered hand are transmitted through contact points. The contact interactions between the object and the multifingered hand generate motion (rolling, sliding and breaking contact points). A dynamic model which can predict the change of contact states for applied joint driving torques of a multi-fingered hand is needed for control simulation [11] or motion planning [13] of manipulation using sliding or rolling. Therefore, it is important to ponder the contact dynamics more in depth and to construct a dynamic model with this in mind. There are considerable studies of control algorithms and also with inverse dynamic problems for object contact motion manipulation, [1], [2], [9], [14]. To my knowledge, there is no study of the forward dynamic problem that takes into account contact mode transition. Our aim is to make manipulation with contact mode transitions by a multifingered hand feasible.

From a theoretical perspective concerning the multibody dynamics, there are various studies about contact dynamics for a general rigid body system. We are able to use the prior knowledge for the compliance and complementarity models which are proposed as a dynamic model for the contact dynamics problem, and express problems for these models.

In the case of the compliance model, each contact is modeled as a contact compliance. It is assumed that the deformations between two bodies generate contact forces. The contact forces are uniquely determined and there is no question of indeterminacy. Some work on twodimensional contact dynamic problems has been reported [4], [5], [6]. However, it is difficult to obtain a contact compliance model which shows a real physical effect properly (particularly in three-dimensional space). The models lead to high-dimensional stiff equations and have a high computational cost.

In the case of the complementarity model, the contact dynamics problem is formulated as the linear complementarity problem (LCP) [3], based on complementary constraints on relative acceleration, $a_{i}$, and the corresponding force, $f_{i}$ at each contact point between rigid bodies. That is, if a contact is maintained, then $a_{i}=0, f_{i} \geq 0$. Similarly, if a contact is breaking, then $a_{i} \geq 0, f_{i}=$ 0 . The complementarity constraints on $a_{i}$ and $f_{i}$ can be expressed by (1) for $n$-contact points. On the other hand, we can derive the linear equation (2) for $a_{i}$ and $f_{i}$ from kinematic and dynamic equations for all multi-rigid bodies.

$$
\begin{aligned}
a_{i} f_{i} & =0, a_{i} \geq 0, f_{i} \geq 0, \text { for } i=1, \cdots n \\
\boldsymbol{a} & =\boldsymbol{A} \boldsymbol{f}+\boldsymbol{b}
\end{aligned}
$$

The square matrix, $\boldsymbol{A} \in \Re^{n \times n}$, and the vector, $\boldsymbol{b} \in \Re^{n}$, is decided for the configuration and dynamic parameters of rigid bodies. The LCP consists of finding vectors, $\boldsymbol{a}$ and $f \in \Re^{n}$ that satisfy (1) and (2) for given matrix, $\boldsymbol{A}$, and vector, $\boldsymbol{b}$. There have been studies of the forward dynamics problem using complementarity models [7], [8], [12]. However, these models are either restricted to two dimensions, or the dynamic validity of the model and its solution is not discussed.

Our objective is to propose a novel complementarity model for a general three-dimensional manipulation system and its algorithm in order to overcome the abovementioned problems. We first formulate the basic equations of kinematics and dynamics for a manipulation system. We then derive the complementarity constraints for rolling contacts by decomposition of frictional properties in the tangential direction. A complementarity model for a three-dimensional manipulation system is then formulated using the obtained complementarity constraints for rolling 


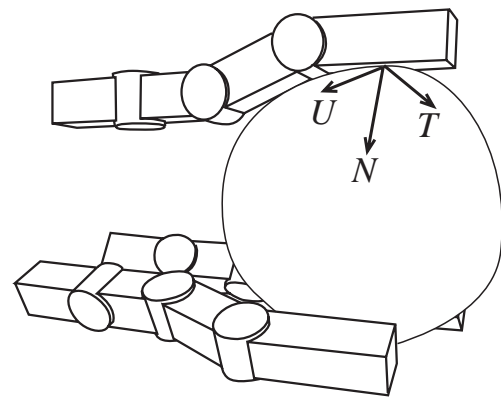

Fig. 1. Multi-fingered hand manipulation.

contacts. We show the simulation algorithm which can compute dynamically determinate solutions. Finally we apply the proposed model to the dynamic simulation of a multi-fingered hand.

\section{RIGID BODY CONTACT MODEL FOR MANIPULATION SYSTEM}

In this section, we present fundamental mathematical models for the rigid body contact problem of the threedimensional manipulation system shown in Fig.1. We assume that an object and a multi-fingered hand are rigid, each link of the hand has at most one contact point with the object, and each joint has one degree of freedom. Also the manipulation system has a configuration that new contact does not become active the instant joint driving torques are applied. Suppose there are $n_{C}$ contacts, consisting of $n_{R}$ rolling contacts and $n_{S}$ sliding contacts. Let the subscripts $R$ and $S$ denote sliding and rolling contacts respectively. A contact frame is assigned to each contact point on the object's surface, with its axes aligned with the inward normal, $N$, and two tangential directions, $T$ and $U$, spanning the tangent plane. We denote the $i$ th contact point by subscript $i$.

\section{A. Coulomb friction}

According to Coulomb's law, a contact force at the $i$ th contact point between the object and the link, as shown in Fig.2, lies within or on the boundary of its friction circular cone represented as:

$$
\sqrt{f_{T i}^{2}+f_{U i}^{2}} \leq \mu f_{N i}
$$

where $f_{N i}, f_{T i}$ and $f_{U i}$ indicate components of contact force in the $N, T$ and $U$ directions expressed in the $i$ th contact frame, and $\mu$ is the coefficient of friction.

Consider the relationship between relative velocities and accelerations, and the corresponding contact forces. Let $\boldsymbol{v}_{i}=\left[\begin{array}{lll}v_{N i}, & v_{T i}, & v_{U i}\end{array}\right]^{T}$ and $\boldsymbol{a}_{i}=\left[\begin{array}{lll}a_{N i}, & a_{T i}, & a_{U i}\end{array}\right]^{T}$ be the relative velocity and acceleration in the $N, T$ and $U$ directions expressed in the $i$ th contact frame. First, we discuss the relationship between them in the normal direction. When the contact will be maintained, we have $a_{N i}=0, f_{N i} \geq 0$. On the other hand, when the contact

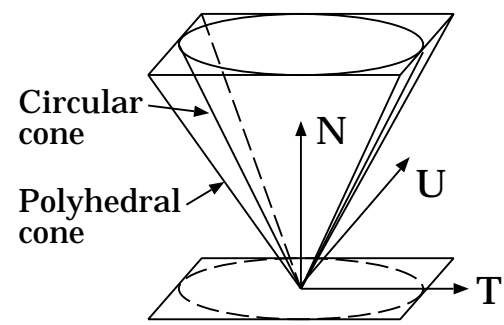

Fig. 2. Friction circular cone and friction polyhedral cone model.

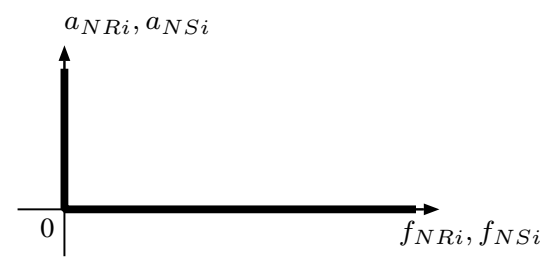

Fig. 3. Complementarity for contact force and relative acceleration in the normal direction.

will break, we have $a_{N i} \geq 0, f_{N i}=0$. The properties of $a_{N i}$ and $f_{N i}$ are called complementary. Since this condition holds for both rolling and sliding contacts, we can get the following complementary conditions:

$$
\left.\begin{array}{r}
a_{N R i} f_{N R i}=0, \quad a_{N R i} \geq 0, f_{N R i} \geq 0 \\
a_{N S i} f_{N S i}=0, a_{N S i} \geq 0, f_{N S i} \geq 0
\end{array}\right\}
$$

Fig.3 illustrates the relation between the normal component of the contact force and the relative acceleration, shown in (4). As you can see from this figure, the complementary constraints can be illustrated by two branches which form a rectangular corner.

Next, we discuss constraints on rolling and sliding contacts in the tangential direction. If a relative velocity is not zero, that is, $v_{T i}^{2}+v_{U i}^{2} \neq 0$, sliding contact is generated. The contact force must lie on the boundary of the friction cone with its tangential component opposite to the relative velocity in the $T$ and $U$ directions:

$$
f_{\bullet S i}=\tilde{\mu}_{\bullet i} f_{N S i}, \quad \text { for } \bullet \in\{T, U\}
$$

where $\widetilde{\mu}_{\bullet i}=\frac{-\mu v_{\bullet}}{\sqrt{\left(v_{T i}\right)^{2}+\left(v_{U i}\right)^{2}}}$. Henceforth, let the subscript - denote the $T$ or $U$ directions.

Conversely, if the relative velocity is zero, that is, $v_{T i}=v_{U i}=0$, the contact becomes rolling. However, when we discuss the feasibility of a transition from rolling to sliding, we should take into consideration a relative acceleration in the tangential direction. When the rolling contact is maintained, the relative acceleration in the tangential direction is zero, i.e., $a_{T R i}=a_{U R i}=0$. In this case, the contact force which has any magnitude and direction lies within the friction cone. When the rolling contact begins to slide, relative acceleration is generated and satisfies $a_{T R i}^{2}+a_{U R i}^{2} \neq 0$. The contact force lies on the boundary of the friction cone with its tangential component opposite to the relative acceleration. 
Summarizing the constraints mentioned above, we get the following constraints between the tangential component of the contact force and the relative acceleration for the rolling contact:

$$
\left.\begin{array}{rll}
\sqrt{f_{T R i}^{2}+f_{U R i}^{2}}<\mu_{i} f_{N R i} & \text { for } & a_{T R i}=a_{U R i}=0 \\
f_{\bullet R i}=+\mu f_{N R i} & \text { for } & a_{\bullet R i}<0 \\
f_{\bullet R i}=-\mu f_{N R i} & \text { for } & a_{\bullet R i}>0
\end{array}\right\}
$$

\section{B. Kinematic constraints}

We are able to now formulate kinematic constraints for the manipulation system with rolling and sliding contacts. As a result, we can define a set of coordinate frames as: The reference frame, $\Sigma_{B}$, is fixed to the hand palm; $\Sigma_{O}$ is fixed to the center of mass of the object; $\Sigma_{i j}$ is a contact frame at the $j$ th contact point of the $i$ th finger, as shown in Fig.1. We define $\boldsymbol{x}_{O}$ and $\boldsymbol{R}_{O}$ to be the position vector and rotational matrix of $\Sigma_{O}$ expressed in $\Sigma_{B}$.

The normal and tangential components, $v_{N i j}, v_{T i j}$ and $v_{U i j}$, of a relative velocity of the $j$ th contact point on the $i$ th finger expressed in $\Sigma_{i j}$ can be given by a difference between the velocity of the $j$ th contact point on the object and the velocity of the $j$ th contact point on the link:

$$
v_{\Sigma i j}=\boldsymbol{G}_{\Sigma i j}^{T} \boldsymbol{v}_{O}-\boldsymbol{J}_{\Sigma i j} \dot{\boldsymbol{\theta}}_{i} \quad \text { for } \quad \Sigma \in\{N, T, U\}
$$

where $G_{N i j}^{T} \in \Re^{1 \times 6}$ is a matrix which maps the object's linear and angular velocity, $\boldsymbol{v}_{O} \in \Re^{6}$, to the normal component of the velocity of the $j$ th contact point on the object. The matrix $\boldsymbol{J}_{N i j} \in \Re^{1 \times n_{\theta i}}$ maps the joint velocity, $\dot{\boldsymbol{\theta}}_{i} \in \Re^{n_{\theta i}}$, of the $i$ th finger to the normal component of the velocity of the $j$ th contact point on the link. Similarly, the matrices $\boldsymbol{G}_{T i j}, \boldsymbol{G}_{U i j}, \boldsymbol{J}_{T i j}$ and $\boldsymbol{J}_{U i j}$ are defined. $n_{\theta i}$ is the number of joints of the $i$ th finger. Henceforth, the subscript $\Sigma$ denotes the $N, T$ and $U$ directions.

The relative motions at the contact point are subject to constraint forces according to the contact modes. The rolling contact constrains the relative velocities in the $N, T$ and $U$ directions to be zero, while the relative velocity in the only normal direction is constrained to be zero by the sliding contact. Summing (7) for all components of constrained relative velocities of rolling and sliding contact points yields

$$
\left[\begin{array}{c}
\boldsymbol{v}_{N S} \\
\boldsymbol{v}_{N R} \\
\boldsymbol{v}_{T R} \\
\boldsymbol{v}_{U R}
\end{array}\right]=\left[\begin{array}{c}
\boldsymbol{W}_{N S}^{T} \\
\boldsymbol{W}_{N R}^{T} \\
\boldsymbol{W}_{T R}^{T} \\
\boldsymbol{W}_{U R}^{T}
\end{array}\right] \boldsymbol{V}
$$

where

$$
\begin{aligned}
& \boldsymbol{W}_{\Sigma R}^{T}=\left[\begin{array}{ll}
\boldsymbol{G}_{\Sigma R}^{T} & -\boldsymbol{J}_{\Sigma R}
\end{array}\right] \in \Re^{n_{R} \times\left(6+n_{\theta}\right)} \\
& \boldsymbol{W}_{\Sigma S}^{T}=\left[\begin{array}{ll}
\boldsymbol{G}_{\Sigma S}^{T} & -\boldsymbol{J}_{\Sigma S}
\end{array}\right] \in \Re^{n_{S} \times\left(6+n_{\theta}\right)}
\end{aligned}
$$

$\boldsymbol{v}_{N S} \in \Re^{n_{S}}$ is formed by the vertical concatenation of the normal components of the relative velocity, $v_{N i j}$ at all sliding contact points. Similarly, $\boldsymbol{v}_{N R}, \boldsymbol{v}_{T R}$ and
$\boldsymbol{v}_{U R}$ are defined. $\boldsymbol{V}=\left[\boldsymbol{v}_{O}^{T}, \dot{\boldsymbol{\theta}}^{T}\right]^{T} \in \Re^{6+n_{\theta}}$ and $\dot{\boldsymbol{\theta}}=\left[\dot{\boldsymbol{\theta}}_{1}^{T}, \dot{\boldsymbol{\theta}}_{2}^{T}, \cdots, \dot{\boldsymbol{\theta}}_{m}^{T}\right]^{T} \in \Re^{n_{\theta}}$. The $n_{\theta}$ is the number of joints. The matrix $\boldsymbol{G}_{N S}^{T} \in \Re^{n_{S} \times 6}$ (resp. $\boldsymbol{J}_{N S} \in \Re^{n_{S} \times n_{\theta}}$ ) is formed by the stack of $\boldsymbol{G}_{N i j}^{T}$ (resp. $\boldsymbol{J}_{N i j}$ ) for the sliding contact points. Similarly, the matrices $G_{\Sigma R}^{T} \in \Re^{n_{R} \times 6}$ and $\boldsymbol{J}_{\Sigma R} \in \Re^{n_{R} \times n_{\theta}}$ are defined for rolling contact points.

Differentiating (8) yields relative accelerations:

$$
\left[\begin{array}{c}
\boldsymbol{a}_{N S} \\
\boldsymbol{a}_{N R} \\
\boldsymbol{a}_{T R} \\
\boldsymbol{a}_{U R}
\end{array}\right]=\left[\begin{array}{c}
\boldsymbol{W}_{N S}^{T} \\
\boldsymbol{W}_{N R}^{T} \\
\boldsymbol{W}_{T R}^{T} \\
\boldsymbol{W}_{U R}^{T}
\end{array}\right] \dot{\boldsymbol{V}}+\left[\begin{array}{c}
\dot{\boldsymbol{W}}_{N S}^{T} \\
\dot{\boldsymbol{W}}_{N R}^{T} \\
\dot{\boldsymbol{W}}_{T}^{T} \\
\dot{\boldsymbol{W}}_{U R}^{T}
\end{array}\right] \boldsymbol{V}
$$

\section{Dynamics}

The dynamic equation of motion of the object can be written, using the matrices $\boldsymbol{G}_{\Sigma R}$ and $\boldsymbol{G}_{\Sigma S}$ in (9) and (10), as follows:

$$
\begin{aligned}
\boldsymbol{M}_{O} \dot{\boldsymbol{v}}_{O}= & \boldsymbol{G}_{N R} \boldsymbol{f}_{N R}+\boldsymbol{G}_{T R} \boldsymbol{f}_{T R}+\boldsymbol{G}_{U R} \boldsymbol{f}_{U R} \\
& +\boldsymbol{G}_{\mu S} \boldsymbol{f}_{N S}+\boldsymbol{h}_{O}
\end{aligned}
$$

where $\boldsymbol{G}_{\mu S}=\boldsymbol{G}_{N S}+\boldsymbol{G}_{T S} \widetilde{\boldsymbol{\mu}}_{T}+\boldsymbol{G}_{U S} \widetilde{\boldsymbol{\mu}}_{U}, \widetilde{\boldsymbol{\mu}}_{\bullet}=$ $\operatorname{diag}\left[\tilde{\mu}_{\bullet 1}, \widetilde{\mu}_{\bullet 2}, \cdots, \widetilde{\mu}_{\bullet n_{S}}\right] \cdot \boldsymbol{f}_{\Sigma R} \in \Re^{n_{R}}$ and $\boldsymbol{f}_{\Sigma S} \in$ $\Re^{n_{S}}$ are contact force vectors for rolling and sliding contact respectively. These vectors are formed by the vertical concatenation of each component of contact force in the $N, T$ and $U$ directions. $M_{O} \in \Re^{6 \times 6}$ is the mass matrix of the object, and $\boldsymbol{h}_{O} \in \Re^{6}$ is the centrifugal, Coriolis and gravitational force vector.

The motion equation of the multi-fingered hand can be written, using the matrices $\boldsymbol{J}_{\Sigma R}$ and $\boldsymbol{J}_{\Sigma S}$ in (9) and (10), as follows:

$$
\begin{aligned}
\boldsymbol{M}_{H} \ddot{\boldsymbol{\theta}}= & \boldsymbol{\tau}-\boldsymbol{J}_{N R}^{T} \boldsymbol{f}_{N R}-\boldsymbol{J}_{T R}^{T} \boldsymbol{f}_{T R}-\boldsymbol{J}_{U R}^{T} \boldsymbol{f}_{U R} \\
& -\boldsymbol{J}_{\mu S}^{T} \boldsymbol{f}_{N S}-\boldsymbol{h}_{H}
\end{aligned}
$$

where $\boldsymbol{J}_{\mu S}^{T}=\boldsymbol{J}_{N S}^{T}+\boldsymbol{J}_{T S}^{T} \widetilde{\boldsymbol{\mu}}_{T}+\boldsymbol{J}_{U S}^{T} \widetilde{\boldsymbol{\mu}}_{U}, \quad \boldsymbol{M}_{H} \in \Re^{n_{\theta} \times n_{\theta}}$ is the inertia matrix of the hand, $\boldsymbol{h}_{H} \in \Re^{6}$ is a centrifugal, Coriolis and gravitational force vector, and $\tau \in \Re^{n_{\theta}}$ is a joint driving torque vector.

Combining (12) and (13) yields the dynamic equation of motion of the manipulation system:

$$
\boldsymbol{M} \dot{\boldsymbol{V}}=\boldsymbol{W}_{\mu}\left[\begin{array}{c}
\boldsymbol{f}_{N S} \\
\boldsymbol{f}_{N R} \\
\boldsymbol{f}_{T R} \\
\boldsymbol{f}_{U R}
\end{array}\right]+\left[\begin{array}{c}
\boldsymbol{h}_{O} \\
\boldsymbol{\tau}-\boldsymbol{h}_{H}
\end{array}\right]
$$

where $\boldsymbol{M}=\operatorname{diag}\left[\boldsymbol{M}_{O}, \boldsymbol{M}_{H}\right], \boldsymbol{W}_{\mu S}=\left[\boldsymbol{G}_{\mu S}^{T},-\boldsymbol{J}_{\mu S}\right]^{T}$, $\boldsymbol{W}_{\mu}=\left[\boldsymbol{W}_{\mu S}, \boldsymbol{W}_{N R}, \boldsymbol{W}_{T R}, \boldsymbol{W}_{U R}\right]$.

Finally, using the obtained model of the manipulation system in this section, we define the rigid-body contact problem for a three-dimensional manipulation system as follows: 
Definition 1: The rigid-body contact problem for a three-dimensional manipulation system is to find $\ddot{\boldsymbol{q}}_{O}, \ddot{\boldsymbol{\theta}}, \boldsymbol{f}_{N R}, \boldsymbol{f}_{T R}, \boldsymbol{f}_{U R}, \boldsymbol{f}_{N S}, \boldsymbol{a}_{N R}, \boldsymbol{a}_{T R}, \boldsymbol{a}_{U R}$ and $\boldsymbol{a}_{N S}$ which satisfy (4), (6), (11) and (14), for given $\boldsymbol{q}_{O}, \dot{\boldsymbol{q}}_{O}, \boldsymbol{M}_{O}, \boldsymbol{h}_{O}, \boldsymbol{\theta}, \dot{\boldsymbol{\theta}}, \boldsymbol{\tau}, \boldsymbol{M}_{H}$ and $\boldsymbol{h}_{H}$, in order to predict the accelerations of the object and multi-fingered hand, contact forces and the transitions of contact modes.

\section{COMPLEMENTARITY FORMULATIONS}

\section{A. Complementarity for rolling contact}

The constraints for rolling contacts in the tangential directions are expressed by (6), which are non-linear constraints for the contact forces, $f_{T R i}, f_{U R i}$ and $f_{N R i}$. In order to facilitate the analysis, we approximate the friction circular cone by a polyhedral cone, as shown in Fig.2. Instead of (6), we can get linear constraints for rolling contacts as:

$$
\left.\begin{array}{rll}
-\mu f_{N R i}<f_{\bullet R i}<+\mu f_{N R i} & \text { for } & a_{\bullet R i}=0 \\
f_{\bullet R i}=+\mu f_{N R i} & \text { for } & a_{\bullet R i}<0 \\
f_{\bullet R i}=-\mu f_{N R i} & \text { for } & a_{\bullet R i}>0
\end{array}\right\}
$$

Fig.4 illustrates the relationship between the contact force and the relative acceleration in the tangential direction, shown in (15). Fig.4 shows the three branches which form two rectangular corners, which is not the same as Fig. 3 that expresses the complementarity constraints. As a result, we can derive complementary constraints for rolling contacts in the tangential directions by changing the shape of Fig.4 with the decomposition of the frictional properties shown in (15).

As shown in Fig.4, the tangential components of the contact force and the relative acceleration belong to one of the following two sets, $S_{1}$ and $S_{2}$, based on the signs of the components as:

$$
\begin{aligned}
& S_{1}=\left\{f_{\bullet R i}, a_{\bullet R i} \mid\right. \\
& \text { (i) } a_{\bullet R i}<0 \text { and } f_{\bullet R i}=+\mu f_{N R i} \text { or } \\
& \text { (ii) } \left.a_{\bullet R i}=0 \text { and } 0 \leq f_{\bullet R i}<\mu f_{N R i}\right\} \\
& S_{2}=\left\{f \bullet R i, a_{\bullet R i} \mid\right. \\
& \text { (i) } a_{\bullet R i}>0 \text { and } f_{\bullet R i}=-\mu f_{N R i} \text { or } \\
& \text { (ii) } \left.a_{\bullet R i}=0 \text { and }-\mu f_{N R i}<f_{\bullet R i} \leq 0\right\}
\end{aligned}
$$

Fig.5(a) illustrates the relation between the tangential components of contact forces and the relative accelerations which belong to the sets, $S_{1}$ and $S_{2}$. This figure corresponds to graphs divided into the left and right parts in Fig.4 symmetrically with respect to the $a_{T R i}, a_{U R i^{-}}$ axis.

Transforming the variables, $f_{\bullet R i}$ and $a_{\bullet R i}$, in (16) into non-negative variables, $f_{\bullet R i}^{+}, f_{\bullet R i}^{-}, a_{\bullet R i}^{+}$and $a_{\bullet R i}^{-} \geq 0$,

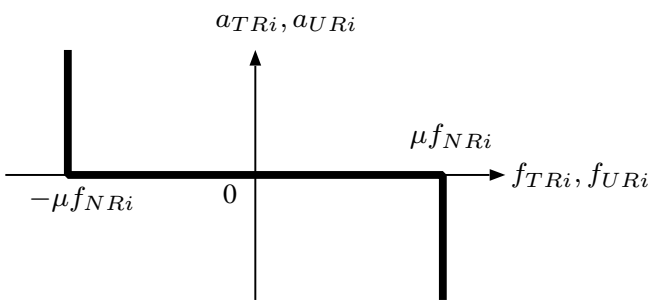

Fig. 4. Relation between contact force and relative acceleration in the tangential direction for friction polyhedral cone model.

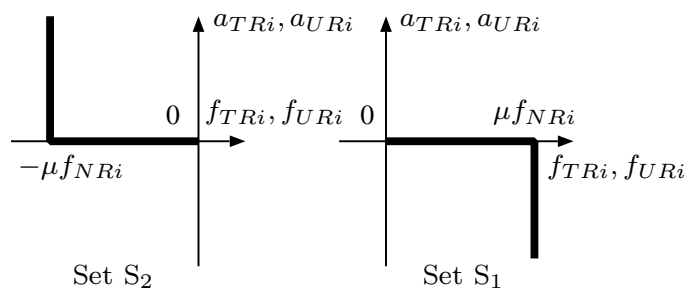

(a)

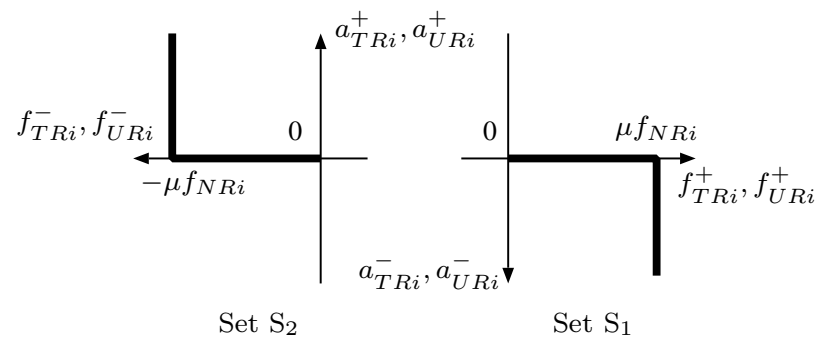

(b)

Fig. 5. Decomposition of tangential properties of rolling contact.

we re-define the sets $S_{1}$ and $S_{2}$ as follows:

$$
\left.\begin{array}{rl}
S_{1}=\{ & f_{\bullet R i}^{+}, a_{\bullet R i}^{-} \mid \\
& (\mathrm{i}) a_{\bullet R i}^{-}>0 \text { and } f_{\bullet R i}^{+}=\mu f_{N R i} \text { or } \\
& \left.(\text { ii }) a_{\bullet R i}^{-}=0 \text { and } 0 \leq f_{\bullet R i}^{+}<\mu f_{N R i}\right\} \\
S_{2}=\{ & f_{\bullet R i}^{-}, a_{\bullet R i}^{+} \mid \\
& (\mathrm{i}) a_{\bullet R i}^{+}>0 \text { and } f_{\bullet R i}^{-}=\mu f_{N R i} \text { or } \\
& \left(\text { ii) } a_{\bullet R i}^{+}=0 \text { and } 0 \leq f_{\bullet R i}^{-}<\mu f_{N R i}\right\}
\end{array}\right\}
$$

where superscripts, + and - , denote that $f \bullet R i$ and $a_{\bullet} R i$ are non-negative and non-positive variables respectively.

Fig.5(b) illustrates the relationship among the nonnegative variables for the redined sets, $S_{1}$ and $S_{2}$. With the decomposition of the frictional properties in the tangential direction, the contact sets, $S_{1}$ and $S_{2}$, can be expressed by the independent variables, $S_{1} \ni\left\{f_{\bullet R i}^{+}, a_{\bullet R i}^{-} \geq 0\right\}$ and $S_{2} \ni\left\{f_{\bullet R i}^{-}, a_{\bullet R i}^{+} \geq 0\right\}$, and we can decompose Fig.4 into 2-two branches with a rectangular corner.

We then introduce non-negative slack variables, $\Delta_{\bullet i}^{+}, \Delta_{\bullet i}^{-} \geq 0$, which satisfy

$$
\left.\begin{array}{ll}
f_{\bullet R i}^{+}+\Delta_{\bullet i}^{+}=\mu f_{N R i}, & \Delta_{\bullet i}^{+} \geq 0 \\
f_{\bullet R i}^{-}+\Delta_{\bullet i}^{-}=\mu f_{N R i}, & \Delta_{\bullet i}^{-} \geq 0
\end{array}\right\}
$$


Substituting $f_{\bullet R i}^{+}, f_{\bullet R i}^{-}$in (18) into (17) yields

$$
\begin{aligned}
& S_{1}=\left\{\Delta_{\bullet}^{+}, a_{\bullet R i}^{-} \mid a_{\bullet R i}^{-} \Delta_{\bullet i}^{+}=0,\right. \\
& \left.a_{\bullet R i}^{-} \geq 0,0 \leq \Delta_{\bullet i}^{+} \leq \mu f_{N R i}\right\} \\
& S_{2}=\left\{\begin{array}{ll}
\Delta_{\bullet i}^{-}, a_{\bullet R i}^{+} \mid & a_{\bullet R i}^{+} \Delta_{\bullet i}^{-}=0, \\
& \left.a_{\bullet R i}^{+} \geq 0,0 \leq \Delta_{\bullet i}^{-} \leq \mu f_{N R i}\right\}
\end{array}\right\}
\end{aligned}
$$

It is clear that (19) represents complementary constraints on both $a_{\bullet R i}^{-}$and $\Delta_{\bullet i}^{+}$, and $a_{\bullet R i}^{+}$and $\Delta_{\bullet}^{-}$. Finally, the complementary constraints for the rolling contact in the tangential direction can be expressed by (19) by introducing the non-negative relative accelerations, $a_{\bullet R i}^{+}, a_{\bullet R i}^{-}$and the non-negative slack variables, $\Delta_{\bullet}^{+}, \Delta_{\bullet}^{-}$.

\section{B. Complementarity model}

We derive a complementarity model for a threedimensional manipulation system from the complementary constraints, (4) and (19), and the kinematic and dynamic equations for the manipulation system, (11) and (14).

First, we derive the complementary constraints expressed by (1) from (4) and (19). Summing (4) for all contact points yields

$$
\left.\begin{array}{lll}
\boldsymbol{a}_{N R} \cdot \boldsymbol{f}_{N R}=0, & \boldsymbol{a}_{N R} \geq \mathbf{0}, & \boldsymbol{f}_{N R} \geq \mathbf{0} \\
\boldsymbol{a}_{N S} \cdot \boldsymbol{f}_{N S}=0, & \boldsymbol{a}_{N S} \geq \mathbf{0}, & \boldsymbol{f}_{N S} \geq \mathbf{0}
\end{array}\right\}
$$

Similarly, summing (19) for all rolling contact points yields

$$
\begin{aligned}
& \left.\boldsymbol{a}_{T R}^{\Pi_{T}} \cdot \boldsymbol{\Delta}_{T}^{\overline{\Pi_{T}}}=0, \quad \boldsymbol{a}_{T R}^{\Pi_{T}} \geq \mathbf{0}, \quad \mathbf{0} \leq \boldsymbol{\Delta}_{T}^{\overline{\Pi_{T}}} \leq \mu \boldsymbol{f}_{N R}\right\} \\
& \left.\boldsymbol{a}_{U R}^{\Pi_{U}} \cdot \boldsymbol{\Delta}_{U}^{\overline{\Pi_{U}}}=0, \quad \boldsymbol{a}_{U R}^{\Pi_{U}} \geq \mathbf{0}, \quad \mathbf{0} \leq \boldsymbol{\Delta}_{U}^{\overline{\Pi_{U}}} \leq \mu \boldsymbol{f}_{N R}\right\}
\end{aligned}
$$

where $\Pi_{\bullet} \in\left\{\{+,-\}, \Pi_{\bullet} \in\left\{\Pi_{\bullet 1}, \Pi_{\bullet 2}, \cdots, \Pi_{\bullet} n_{R}\right\}\right.$. The inverse sign of $\Pi_{T i}, \Pi_{U i}$ is expressed by $\bar{\Pi}_{T i}, \bar{\Pi}_{U i}$.

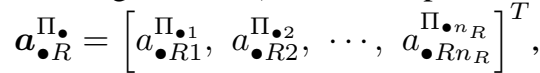

$$
\begin{aligned}
& \Delta_{\bullet}^{\Pi_{\bullet}}=\left[\Delta_{\bullet 1}^{\Pi_{\bullet} 1}, \Delta_{\bullet 2}^{\Pi_{\bullet} 2}, \cdots, \Delta_{\bullet}^{\Pi_{\bullet} n_{R}}\right]^{T} .
\end{aligned}
$$

Combining (20) and (21) yields the complementary constraints which corresponds to (1) as follows:

$$
\left[\begin{array}{c}
\boldsymbol{a}_{N S} \\
\boldsymbol{a}_{N R} \\
\boldsymbol{a}_{T R}^{\Pi_{T}} \\
\boldsymbol{a}_{U R}^{\Pi_{U}}
\end{array}\right]^{T}\left[\begin{array}{c}
\boldsymbol{f}_{N S} \\
\boldsymbol{f}_{N R} \\
\boldsymbol{\Delta}_{T} \\
\boldsymbol{\Delta}_{U}^{\frac{\Pi_{T}}{\Pi_{U}}}
\end{array}\right]=0,\left[\begin{array}{c}
\boldsymbol{a}_{N S} \\
\boldsymbol{a}_{N R} \\
\boldsymbol{a}_{T R}^{\Pi_{T}} \\
\boldsymbol{a}_{U R}^{\Pi_{U}}
\end{array}\right] \geq \mathbf{0},\left[\begin{array}{c}
\boldsymbol{f}_{N S} \\
\boldsymbol{f}_{N R} \\
\boldsymbol{\Delta}_{T} \\
\boldsymbol{\Delta}_{U}^{\frac{T}{\Pi_{U}}}
\end{array}\right] \geq \mathbf{0}
$$

where

$$
\boldsymbol{\Delta}_{T}^{\overline{\Pi_{T}}} \leq \mu \boldsymbol{f}_{N R}, \quad \boldsymbol{\Delta}_{U}^{\overline{\Pi_{U}}} \leq \mu \boldsymbol{f}_{N R}
$$

Next, we derive the linear equation expressed by (2) from (11) and (14). To begin with, we rewrite (11) and (14) using non-negative variables $\left(f_{N R i}, f_{N S i}, a_{N R i}, a_{N S i}, a_{\bullet R i}^{+}, a_{\bullet R i}^{-}, \Delta_{\bullet}^{+}\right.$, and $\left.\Delta_{\bullet i}^{-}\right)$, which are introduced in (4) and (19).

Each tangential contact force, $f_{T R i}$ and $f_{U R i}$, at the rolling contact belongs to either contact sets $S_{1}$ or $S_{2}$ in (17). Therefore, we may rewrite (14) using the nonnegative variables, $f_{T R i}^{+}, f_{T R i}^{-}, f_{U R i}^{+}$and $f_{U R i}^{-}$, instead of $f_{T R i}$ and $f_{U R i}$. Then, we may solve for the variables, $f_{T R i}^{ \pm}$and $f_{U R i}^{ \pm}$in (18) and substitute them into (14). This yields

$$
\boldsymbol{M} \dot{\boldsymbol{V}}=\boldsymbol{W}_{\mu\left(\Pi_{T}, \Pi_{U}\right)}\left[\begin{array}{c}
\boldsymbol{f}_{N S} \\
\boldsymbol{f}_{N R} \\
\boldsymbol{\Delta}_{T}^{\Pi_{T}} \\
\boldsymbol{\Delta}_{U}^{\Pi_{U}}
\end{array}\right]+\left[\begin{array}{c}
\boldsymbol{h}_{O} \\
\boldsymbol{\tau}-\boldsymbol{h}_{H}
\end{array}\right]
$$

where

$$
\begin{aligned}
& \boldsymbol{W}_{\bullet R i}^{+}=\boldsymbol{W}_{\bullet R i}, \quad \boldsymbol{W}_{\bullet R i}^{-}=-\boldsymbol{W}_{\bullet R i}, \\
& \boldsymbol{W}_{\bullet}^{\Pi \cdot \bullet}=\left[\boldsymbol{W}_{\bullet R 1}^{\Pi \Pi_{\bullet}}, \boldsymbol{W}_{\bullet R 2}^{\Pi_{\bullet 2}}, \cdots, \boldsymbol{W}_{\bullet R n_{R}}^{\Pi_{\bullet_{n}}}\right], \\
& \boldsymbol{W}_{\mu\left(\Pi_{T}, \Pi_{U}\right)}=\left[\boldsymbol{W}_{\mu S}, \boldsymbol{W}_{N R}+\mu\left(\boldsymbol{W}_{T R}^{\Pi_{T}}+\boldsymbol{W}_{U R}^{\Pi_{U}}\right),\right. \\
& \left.-\boldsymbol{W}_{T R}^{\Pi_{T}},-\boldsymbol{W}_{U R}^{\Pi_{U}}\right] .
\end{aligned}
$$

Similarly, we may rewrite (11) using the non-negative variables, $a_{T R i}^{+}, a_{T R i}^{-}, a_{U R i}^{+}$and $a_{U R i}^{-}$, instead of $a_{T R i}$ and $a_{U R i}$ as follows:

$$
\left[\begin{array}{c}
\boldsymbol{a}_{N S} \\
\boldsymbol{a}_{N R} \\
\boldsymbol{a}_{T R}^{\Pi_{T}} \\
\boldsymbol{a}_{U R}^{\Pi_{U}}
\end{array}\right]=\boldsymbol{W}_{\left(\Pi_{T}, \Pi_{U}\right)}^{T} \dot{\boldsymbol{V}}+\dot{\boldsymbol{W}}_{\left(\Pi_{T}, \Pi_{U}\right)}^{T} \boldsymbol{V}
$$

where $\boldsymbol{W}_{\left(\Pi_{T}, \Pi_{U}\right)}=\left[\boldsymbol{W}_{N S}, \boldsymbol{W}_{N R}, \boldsymbol{W}_{T R}^{\Pi_{T}}, \boldsymbol{W}_{U R}^{\Pi_{U}}\right]$.

We may solve for the variable, $\dot{\boldsymbol{V}}$, in (24) and substitute it into (25); this yields the linear equation which corresponds to (2) as follows:

$$
\left[\begin{array}{c}
\boldsymbol{a}_{N S} \\
\boldsymbol{a}_{N R} \\
\boldsymbol{a}_{T R}^{\Pi_{T}} \\
\boldsymbol{a}_{U R}^{\Pi_{U}}
\end{array}\right]=\boldsymbol{A}\left[\begin{array}{c}
\boldsymbol{f}_{N S} \\
\boldsymbol{f}_{N R} \\
\boldsymbol{\Delta}_{T}^{\frac{\Pi_{T}}{\Pi_{T}}} \\
\boldsymbol{\Delta}_{U}^{\frac{\Pi_{U}}{\Pi_{U}}}
\end{array}\right]+\boldsymbol{b}
$$

where

$$
\begin{aligned}
& \boldsymbol{A}=\boldsymbol{W}_{\left(\Pi_{T}, \Pi_{U}\right)}^{T} \boldsymbol{M}^{-1} \boldsymbol{W}_{\mu\left(\overline{\Pi_{T}}, \overline{\Pi_{U}}\right)} \\
& \boldsymbol{b}=\boldsymbol{W}_{\left(\Pi_{T}, \Pi_{U}\right)}^{T} \boldsymbol{M}^{-1}\left[\begin{array}{c}
\boldsymbol{h}_{O} \\
\boldsymbol{\tau}-\boldsymbol{h}_{H}
\end{array}\right]+\dot{\boldsymbol{W}}_{\left(\Pi_{T}, \Pi_{U}\right)}^{T} \boldsymbol{V}
\end{aligned}
$$

Finally, we can get the complementarity model which can be expressed by (22) and (26) with constraint (23).

\section{Dynamic simulation algorithm}

A manipulation model is said to be dynamically determinate when we can uniquely predict accelerations, contact forces and contact mode transitions for a given joint driving torque. The manipulation system is required to be dynamically determinate at each instant in order to simulate dynamics for the system. In the proposed complementarity model, we should consider not only that the combinations of feasible contact sets are unknown, but that the LCP does not always have a unique solution in general[3]. In this section, we discuss the dynamic simulation algorithm which can compute the solutions which guarantee the manipulation system to be dynamically determinate. 
The tangential components of the contact force and relative acceleration at each rolling contact point belong to one of the contact set, $S_{1}$ and $S_{2}$. Therefore, we must consider four kinds of combinations of contact sets, $\left(\Pi_{T i}, \Pi_{U i}\right)=\{(+,+),(+,-),(-,+),(-,-)\}$, for each rolling contact point. If the manipulation model is dynamically determinate, then the unique combination of contact set which is mechanically feasible exists. Accordingly, we first solve the LCP in (22), (23) and (26) for all kinds of combinations of contact sets. Then we check whether the feasible solution for accelerations, contact forces and contact mode transitions exists for a unique combination within a contact set. When such a unique combination exists, we then assign the combination as a candidate for a mechanically feasible contact set.

It should be noted that the solution derived from the LCP is not always unique. The LCP in (1) and (2) has a unique solution, $\boldsymbol{a}$ and $\boldsymbol{f}$, for all $\boldsymbol{b}$ if and only if the matrix $\boldsymbol{A}$ is a P-matrix[3]. Since the matrix $\boldsymbol{A}$ in (27) depends on the system configuration, contact modes and dynamic parameters at each instant, we can see that the complementarity model does not always have a unique solution. We can apply the above theory to (27) after we decide the candidate for a mechanically feasible contact set as stated above. If the matrix $\boldsymbol{A}$ for the complementarity model is a P-matrix, then the feasible solution for the LCP are unique solutions. In this way, we can compute the solutions which guarantee the manipulation system to be dynamically determinate.

Now, we can calculate the matrix $\boldsymbol{A}$ in (27) only if the states of the manipulation system at each instant and dynamic parameters are known. The vector $\boldsymbol{b}$ in (28) can be obtained by giving the values of joint driving torques. Therefore, solving (22), (23) and (26) by using Lemke's algorithm[3], we can compute the relative accelerations and contact forces. In addition, we can predict the following five types of contact mode transitions from the relative accelerations, $a_{N S i}, a_{N R i}, a_{T R i}$ and $a_{U R i}$.

(a) Rolling contact

(a1) Rolling contact $\longrightarrow$ Rolling contact $a_{N R i}=a_{T R i}=a_{U R i}=0$

(a2) Rolling contact $\longrightarrow$ Sliding contact $a_{N R i}=0, a_{T R i}^{2}+a_{U R i}^{2} \neq 0$

(b) Sliding contact

(a3) Rolling contact $\longrightarrow$ Breaking contact $a_{N R i} \neq 0$

(b1) Sliding contact $\longrightarrow$ Sliding contact $a_{N S i}=0$

(b2) Sliding contact $\longrightarrow$ Breaking contact $a_{N S i} \neq 0$

\section{COMPUTER SIMUlation}

In this section, when we apply the proposed complementarity model to the dynamic analysis of manipulation by a multi-fingered hand, we show the validity of the

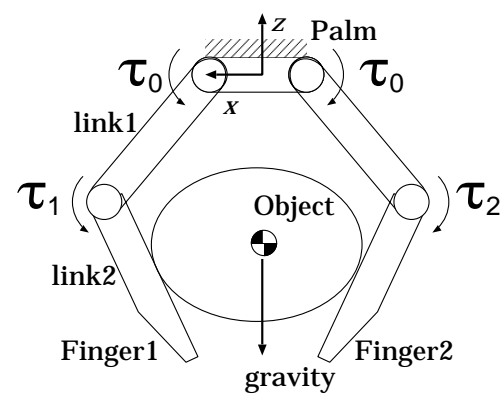

Fig. 6. Simulation model.

model. In addition, we show how the transition of contact modes varies according to the magnitudes of the friction coefficient and the joint driving torques by a computer simulation.

For the sake of simplicity, we now consider a twodimensional manipulation system in a vertical plane, which consists of an elliptical object and a two 2-DOF fingered hand as shown in Fig.6. The position of the object center of mass lies on the $z$ axis of the base frame attached to the palm. The major axis of the object parallels the $x$ axes of the base frame. The object at rest has contact with each 2 nd link of both fingers at the initial time. Therefore, each contact satisfies the frictional constraints for rolling contact. The object has a major axis of $0.37 \mathrm{~m}$ and its minor axis is $0.3 \mathrm{~m}$. The mass of the object is $3.0 \mathrm{~kg}$. The length of each link is $0.3 \mathrm{~m}$ and its mass is $0.5 \mathrm{~kg}$. The fixed palm of the hand is $0.2 \mathrm{~m}$ long.

We then investigate the occurrence of contact mode transition for applied joint driving torques, $\tau_{1}$ and $\tau_{2}$ in the directions shown in Fig.6. A fixed joint driving torque, $\tau_{0}=2.5 \mathrm{Nm}$, is assigned to each of the 1 st joint of both fingers. Fig.7(a),(b) shows the simulation results of the contact mode transitions in $\tau$-space for the coefficient of friction, $\mu=0.2$ and 0.4 respectively. In these figures, the $R S$ indicates that each contact mode at the contact point of the fingers 1 and 2 changes into rolling contact and sliding contact, respectively. The $R R, S R$ and $S S$ are defined similarly. It is clear from our graphical technique by $\tau$-space, that (i) the contact mode changes according to the magnitude of the applied joint driving torque, (ii) the region of rolling contact expands as the coefficient of friction increases. Since the contact points of this manipulation system are arranged symmetrically, the contact mode regions in $\tau$-space are also symmetry. Fig.8(a) shows the regions of the signs of object acceleration in the $z$ direction generated for applied joint driving torques for the case of Fig.7(b). Similarly, Fig.8(b) shows the object acceleration in the $z$ direction generated by the joint driving torques, $\tau_{1}=\tau_{2}$. From these figures, we can see that the object acceleration in the $+z$ direction is generated as the joint driving torques increases. 

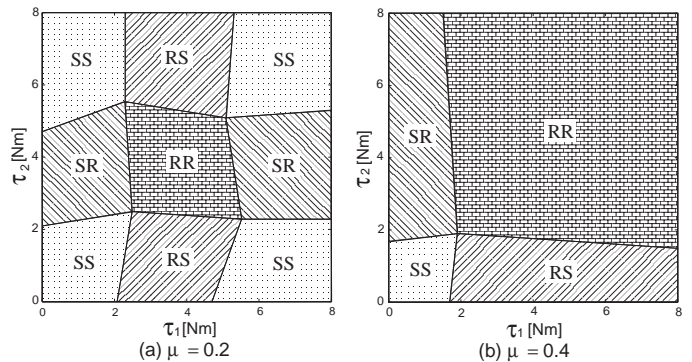

Fig. 7. $\tau$-space for $\mu=0.2$ and 0.4 .

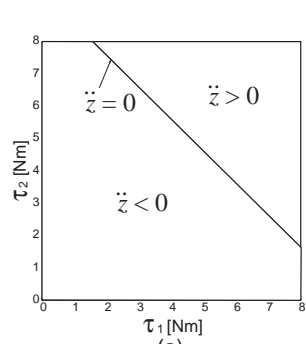

(a)

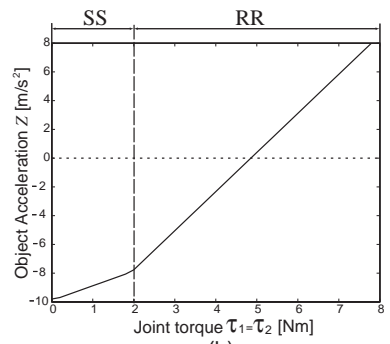

(b)
Fig. 8. Relationship between joint torques and object acceleration in the $z$ direction for $\mu=0.4$.

\section{CONCLUSiON}

In this paper, we have proposed a complementarity model for a general three-dimensional manipulation system with contact mode transitions and its simulation algorithm which can compute the solutions which guarantee the manipulation system to be dynamically determinate. As a result, simulation examples for manipulation by a multi-fingered hand showed the validity of the complementarity model.

In order to actually use the proposed complementarity model for controlling robot hand manipulation with contact mode transitions, we require development of the tactile sensor which can precisely detect contact states. When the complementarity model is not dynamically determinate, the solutions do not have a physical meaning. In order to overcome these problems, we can utilize the algorithm proposed by authors [10], which integrates a compliant model and the complementarity model. This is done by using the complementarity model whenever possible and by switching to a compliant model when the complementarity model has a problem with dynamically determinateness. The proposed algorithm based on the complementarity model can be applied to the computation of a human figure, computer graphic animation and virtual reality, in addition to the problem of multi-fingered manipulation.

\section{REFERENCES}

[1] A.A. Cole, J.E. Hauser and S.S. Sastry, "Kinematics and control of multifingered hands with rolling contac", IEEE Transactions on Aotomatical Control, vol. 34, no. 4, 1989, pp. 398-404.

[2] A.A. Cole, P. Hsu and S.S. Sastry, "Dynamic control of sliding by robot hands for regrasping", IEEE Transactions on Robotics and Automation, vol. 8, no. 1, 1992, pp. 42-52.

[3] R.W.Cottle, J.Pang, R.E.Stone, The Linear Complementarity Problem, Academic Press, 1992.

[4] H.S. Howard and V. Kumar, "A Miminum Principle for the Dynamic Analysis of Systems with Frictional Contacts", In Proc. of IEEE Int. Conf. on Robotics and Automation, 1993, pp. 437-442.

[5] K.H. Hunt and F.R.E. Crossley, "Coefficient of restitution interpreted as damping in vibroimpact", $A S M E$ Journal of Applied Mechanics, vol. 42, E, 1975, pp. 440-445.

[6] P.R. Kraus, A. Fredriksson and V. Kumar, "Modeling of Frictional Contacts for Dynamic Simulation", IROS 1997 Workshop on Dynamic Simulation: Methods and Applications, 1997.

[7] P. Lötstedt, "Coulomb friction in two-dimensional rigid body systems", Zeitschrift Für Angewandte Mathematik und Mechanik, vol. 61, 1981, pp. 605615.

[8] F. Pfeiffer and C. Glocker, Multibody Dynamics with Unilateral Contacts, John Wiley \& Sons (Wiley Series in Nonlinear Science), 1996.

[9] N. Sarker, X. Yun and V. Kumar, "Dynamic Control of 3-D Rolling Contacts in Two-Arm Manipulation", IEEE Transactions on Robotics and Automation, vol. 13, no. 3, 1997, pp. 364-376.

[10] P. Song, M. Yashima, V. Kumar, "Dynamic Simulation for Grasping and Whole Arm Manipulation", IEEE International Conference on Robotics and Automation, 2000, pp. 1082-1087.

[11] P. Song, M. Yashima, V. Kumar, "Dynamics and Control of Whole Arm Grasps", IEEE International Conference on Robotics and Automation, 2001, pp. 2229-2234.

[12] J.C. Trinkle, J.S. Pang, S. Sudarsky, G. Lox, “On dynamics multi-rigid-body contact problems with Coulomb friction", Zeitschrift Für Angewandte Mathematik und Mechanik, vol. 77, no. 4, 1997, pp. 267-279.

[13] M. Yashima and H. Yamaguchi, "Dynamic Motion Planning Whole Arm Grasp Systems Based on Switching Contact Modes", In Proc. of IEEE Int. Conf. on Robotics and Automation, 2002, pp. 24922499.

[14] X. Zheng, N. Nakashima and T. Yoshikawa, "On Dynamic Conrol of Finger Sliding and Object Motion in Manipulation with Multifingered Hands", IEEE Transactions on Robotics and Automation, vol. 16, no. 5, 2000, pp. 469-481. 